\title{
Diffusion measurement from observed transverse beam echoes
}

\author{
Tanaji Sen* \\ Accelerator Physics Center, Fermi National Accelerator Laboratory, Batavia, Illinois 60510, USA \\ Wolfram Fischer \\ Brookhaven National Laboratory, Upton, New York 11973, USA
}

(Received 10 October 2016; published 9 January 2017)

\begin{abstract}
We study the measurement of transverse diffusion through beam echoes. We revisit earlier observations of echoes in the Relativistic Heavy Ion Collider and apply an updated theoretical model to these measurements. We consider three possible models for the diffusion coefficient and show that only one is consistent with measured echo amplitudes and pulse widths. This model allows us to parameterize the diffusion coefficients as functions of the bunch charge. We demonstrate that echoes can be used to measure diffusion much quicker than present methods and could be useful to a variety of hadron synchrotrons.
\end{abstract}

DOI: 10.1103/PhysRevAccelBeams.20.011001

\section{INTRODUCTION}

Beam diffusion can lead to emittance growth, halo formation, and particle loss. A standard method currently used to measure transverse diffusion requires scraping the beam with collimator jaws moved close to the beam, then retracting the jaws, and waiting for the beam to diffuse to the outer position of the jaws [1-5]. This procedure is time consuming, and the method is applicable only to storage rings where the beam circulates for times long enough to enable the measurement. Beam echoes were introduced into accelerator physics more than two decades ago [6,7] and then shown to be useful as a novel method to measure transverse diffusion [8]. A single echo observation can be done typically within a thousand turns with nonlinear tune spreads in the range $0.001-0.01$. Hence, diffusion measurements with echoes would be considerably faster than the standard method and could also enable diffusion to be measured in synchrotrons where beams circulate for relatively short times.

Shortly after the introduction of the beam echo concept, longitudinal unbunched beam echoes were observed at the Fermilab Antiproton Accumulator [9] and then at the CERN Super Proton Synchrotron (SPS) [10]. The original motivation, however, had been to measure transverse diffusion from transverse echoes. In the year 2000, transverse bunched beam echoes were observed in the SPS with two consecutive dipole kicks [11], but no diffusion coefficients were extracted. Later, in 2004-2005, an extensive set of dedicated experiments was carried out at the

\footnotetext{
tsen@fnal.gov

Published by the American Physical Society under the terms of the Creative Commons Attribution 3.0 License. Further distribution of this work must maintain attribution to the author $(s)$ and the published article's title, journal citation, and DOI.
}

Relativistic Heavy Ion Collider (RHIC) with dipole and quadrupole kicks [12], and these will be the focus in this paper. The existing model as applied to the data did not yield consistent values for the diffusion coefficients [13].

The next generation of intensity frontier hadron synchrotrons will require tight control of particle amplitude growth. At Fermilab, the Integrable Optics Test Accelerator (IOTA) [14] ring is under construction where the novel concept of nonlinearly integrable lattices will be tested and could serve as a model for future synchrotrons. This ring offers the opportunity of testing a fast diffusion measurement technique which could help determine the degree of integrability (or stable motion) among different lattice models. With this motivation, we revisit the earlier RHIC measurements with an updated theoretical model to enable extraction of self-consistent diffusion coefficients. In Sec. II, we describe the updated model; in Sec. III, we apply this model to the RHIC data; in Sec. IV, we consider beam-related time scales; and we summarize in Sec. V with lessons to be applied to future echo measurements.

\section{ECHO PULSE WITH DIFFUSION}

The basic beam-echo-generating mechanism is well known. If at some initial time the beam is kicked away from the central orbit, the beam centroid will decohere due to a nonlinear spread of frequencies. If subsequently a quadrupole kick is applied after the centroid response has decayed away, a diminished coherent response will reappear after a time interval equal to the delay between the dipole and quadrupole kicks. Figure 5 in Sec. III C shows an example of this echo formation during the measurements at the RHIC.

Here we discuss the model to calculate the echo amplitude with diffusion using the same method and notation as in Ref. [15]. The phase space coordinates used $x, p$ and action angle coordinates $J, \phi$ are related as 


$$
\begin{gathered}
x=\sqrt{2 \beta J} \cos \phi, \quad p=\alpha x+\beta x^{\prime}=-\sqrt{2 \beta J} \sin \phi, \\
J=\frac{1}{2 \beta}\left(x^{2}+p^{2}\right), \quad \tan \phi=-\frac{p}{x} .
\end{gathered}
$$

The initial distribution is taken to be exponential in the action

$$
\psi_{0}(J)=\frac{1}{2 \pi J_{0}} \exp \left[-\frac{J}{J_{0}}\right]
$$

where $J_{0}=\varepsilon_{0}$, the initial rms emittance.

We first consider the dipole moment after a dipole kick and the general case where the dipole kicker is at a nonzero phase advance from the beam position monitor (BPM) location where the centroid is measured. Following the procedure in Ref. [15], the dipole moment after the dipole kick by an angle $\theta$ is

$$
\langle x\rangle^{\mathrm{amp}}(t)=\frac{\theta \sqrt{\beta_{K} \beta}}{\left(1+\Theta^{2}\right)} \exp \left[-\frac{\beta_{K} \theta^{2}}{2 J_{0}} \frac{\Theta^{2}}{1+\Theta^{2}}\right],
$$

where $\beta_{K}$ and $\beta$ are the beta functions at the kicker and $\mathrm{BPM}$, respectively, and $\Theta=\omega^{\prime} J_{0} t$ with $\omega^{\prime} \equiv d \omega / d J$ the constant slope of the betatron angular frequency with action. This moment is independent of the phase advance from the kicker to the BPM. It differs from the expression in Ref. [15] only by the replacement of $\beta$ by the geometric mean $\beta_{G}=\sqrt{\beta_{K} \beta}$ and $\beta$ in the exponent replaced by $\beta_{K}$. Following the dipole kick, the beam decoheres with the centroid amplitude decaying over a characteristic time $\tau_{D}=1 /\left(\omega^{\prime} J_{0}\right)$, the decoherence time. At time $\tau \gg \tau_{D}$ after the dipole kick, a single turn quadrupole kick is applied to generate the echoes, the first of which occurs around time $2 \tau$. The echo amplitude and pulse shape are affected by the diffusive beam motion. We consider the density distribution to evolve according to the conventional form of the diffusion equation

$$
\frac{\partial}{\partial t} \psi=\frac{\partial}{\partial J}\left[D(J) \frac{\partial}{\partial J}\right] \psi
$$

Here the diffusion coefficient $D(J)$ has the usual dimension of [action ${ }^{2} /$ time], and it differs from the definition of $D(J)$ used in Refs. [8,15]. The treatment in Ref. [15] had developed the theory of the echo response to first order in the quadrupole kick strength. Since the experiments reported in Ref. [12] had observed a linear increase of the echo amplitude with quadrupole strength, this theory should suffice to discuss these experiments. We note that the theory developed earlier in Ref. [7] was nonlinear in this strength parameter. Using the method of Ref. [15], we find that the echo amplitude near time $t>2 \tau$ is

$$
\begin{aligned}
\langle x\rangle(t)= & -\pi \beta_{K} \theta q \tau \int d J \omega^{\prime} J^{2} \psi_{0}^{\prime} \exp \left[-\frac{1}{3} D(J)\left(\omega^{\prime}\right)^{2} t_{1}^{3}\right] \\
& \times \sin [\omega(t-2 \tau)],
\end{aligned}
$$

where $q$ is the dimensionless quadrupole kick strength defined as $q=\beta_{Q} / f$, the ratio of the beta function at the quadrupole to its focal length, and we defined $t_{1}^{3}=(t-\tau)^{3}+$ $\tau^{3}$. We consider the action-dependent transverse angular frequency to be of the form $\omega(J)=\omega_{\beta}+\omega^{\prime} J$, where $\omega_{\beta}$ is the angular betatron frequency and we consider the diffusion coefficient to be of the form

$$
D(J)=\sum_{n=0} D_{n}\left(\frac{J}{J_{0}}\right)^{n},
$$

where all coefficients $D_{n}$ have the same dimensions. The average dipole moment is given by

$$
\begin{aligned}
\langle x\rangle(t)= & \frac{1}{2} \beta_{K} \theta q \mu \tau \omega_{\text {rev }} \exp \left[-\frac{1}{3} D_{0}\left(\omega^{\prime}\right)^{2} t_{1}^{3}\right] \\
& \times \operatorname{Im}\left[e^{\left[i \Phi_{0}\right]} \int_{0}^{\infty} z^{2} \exp \left[-z-\frac{1}{3}\left(\omega^{\prime}\right)^{2} t_{1}^{3} \sum_{n} D_{n} z^{n}\right]\right. \\
& \left.\times e^{\left[i \Phi_{1} J_{0} z\right]} d z\right]
\end{aligned}
$$

where $\omega_{\text {rev }}$ is the angular revolution frequency, $\Phi_{0}=$ $\omega_{\beta}(t-2 \tau)$ and $\Phi_{1}=\omega^{\prime}(t-2 \tau)$. Using $\omega^{\prime}=\left(\omega_{\text {rev }} / \varepsilon\right) \mu$, where $\mu=\nu(\varepsilon)-\nu_{\beta}$ is the tune shift (from the bare tune $\nu_{\beta}$ ) at an action equal to the emittance, it is convenient to define scaled diffusion coefficients $d_{n}$ as

$$
d_{n}=\frac{2}{3} D_{n}\left(\frac{\omega_{\mathrm{rev}}}{\varepsilon}\right)^{2} .
$$

These coefficients $d_{n}$ have the dimension of time ${ }^{-3}$. In the following, we will consider specific cases of the above general form of $D(J)$.

Different physical processes contribute to the diffusion coefficients $D_{n}$. It is likely that space charge effects, beambeam interactions (not present in the RHIC measurements discussed below), and intrabeam scattering all contribute to $D_{0}$ and higher-order coefficients. Early studies at the Tevatron at injection energy [16] with additional sextupoles as the driving nonlinearity had measured a constant $D_{0}$ term which varied with the proximity to a fifth-order resonance. Measurements at the LHC at top energy during collisions showed that diffusion at the smallest amplitude measurable was finite [4], implying a nonzero $D_{0}$. A numerical simulation [17] showed that modulation diffusion leads to a constant diffusion term. Beam-gas scattering and noise in dipoles lead to a $D_{1}$ term, while noise in quadrupoles leads to a $D_{2}$ term. There are likely other sources for these coefficients. Given that the beam is subject to multiple effects, the complete action dependence of the diffusion may be complex. Here we focus on the three simplest models with two diffusion coefficients that can be compared to measurements. 
In the first case, we assume that the diffusion is of the form

$$
D(J)=D_{0}+D_{1}\left(\frac{J}{J_{0}}\right) ;
$$

in this case, the dipole moment is given by

$$
\begin{aligned}
\langle x\rangle(t)= & \beta_{K} \theta q \omega^{\prime} \tau J_{0} \exp \left[-\frac{1}{2} d_{0} \mu^{2} t_{1}^{3}\right] \\
& \times \frac{\left[\left(3 \alpha^{2}-\xi^{2}\right) \xi \cos \Phi_{0}+\left(\alpha^{2}-3 \xi^{2}\right) \alpha \sin \Phi_{0}\right]}{\left(\alpha^{2}+\xi^{2}\right)^{3}}, \\
t_{1}^{3}= & (t-\tau)^{3}+\tau^{3}, \quad \Phi_{0}=\omega_{\beta}(t-2 \tau), \\
\alpha= & 1+\frac{1}{2} d_{1} \mu^{2} t_{1}^{3}, \quad \xi=\omega_{\text {rev }} \mu(t-2 \tau) .
\end{aligned}
$$

The second case is the quadratic dependence model where

$$
D(J)=D_{0}+D_{2}\left(\frac{J}{J_{0}}\right)^{2} .
$$

The general time-dependent form of the echo at time $t=2 \tau+\Delta t$ where $\Delta t$ can have either sign is

$$
\begin{aligned}
\langle x(t)\rangle^{\mathrm{amp}}= & \frac{1}{2} \beta_{K} \theta q \omega_{\mathrm{rev}} \mu \tau \exp \left[-\frac{1}{2} d_{0} \mu^{2} t_{1}^{3}\right] \operatorname{Im}\left[e^{i \Phi_{0}} H_{02}\right], \\
H_{02}(\Delta t) \equiv & \int_{0}^{\infty} z^{2} \exp \left[-a_{0} z-b_{2} z^{2}\right] d z \\
= & \frac{1}{8}\left(\frac{1}{b_{2}}\right)^{5 / 2}\left\{\sqrt{\pi}\left[a_{0}^{2}+2 b_{2}\right] \exp \left(\frac{a_{0}^{2}}{4 b_{2}}\right)\right. \\
& \left.\times \operatorname{Erfc}\left(\frac{a_{0}}{\sqrt{2 b_{2}}}\right)-a_{0} \sqrt{2 b_{2}}\right\} \\
a_{0}= & 1-i \xi=1-i \omega_{\text {rev }} \mu \Delta t, \\
b_{2}= & \frac{1}{2} d_{2} \mu^{2} t_{1}^{3}=\frac{1}{2} d_{2} \mu^{2}\left[(\tau+\Delta t)^{3}+\tau^{3}\right] .
\end{aligned}
$$

Here Erfc is the complementary error function.

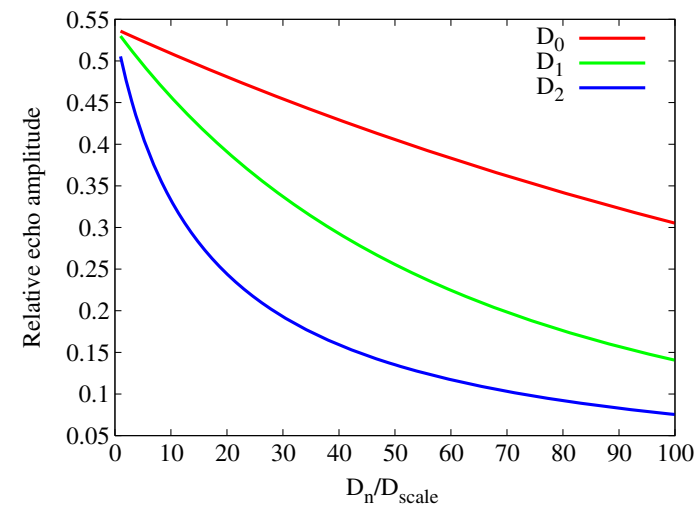

The last case we consider is the linear and quadratic dependence

$$
D(J)=D_{1}\left(\frac{J}{J_{0}}\right)+D_{2}\left(\frac{J}{J_{0}}\right)^{2}
$$

In this case, the time-dependent form of the echo at time $t=2 \tau+\Delta t$ is

$$
\begin{aligned}
\langle x(t)\rangle^{\mathrm{amp}}=\frac{1}{2} \beta_{K} \theta q \omega_{\mathrm{rev}} \mu \tau \operatorname{Im}\left[e^{i \Phi_{0}} H_{12}(\Delta t)\right], \\
H_{12}(\Delta t) \equiv \int_{0}^{\infty} z^{2} \exp \left[-a_{1} z-b_{2} z^{2}\right] d z \\
=\frac{1}{8}\left(\frac{1}{b_{2}}\right)^{5 / 2}\left\{\sqrt{\pi}\left[a_{1}^{2}+2 b_{2}\right] \exp \left(\frac{a_{1}^{2}}{4 b_{2}}\right)\right. \\
\\
\left.\times \operatorname{Erfc}\left(\frac{a_{1}}{\sqrt{2 b_{2}}}\right)-a_{1} \sqrt{2 b_{2}}\right\}, \\
a_{1}=\left(1+b_{1}\right)-i \xi \\
b_{1}=\frac{1}{2} d_{1} \mu^{2} t_{1}^{3}=\frac{1}{2} d_{1} \mu^{2}\left[(\tau+\Delta t)^{3}+\tau^{3}\right] .
\end{aligned}
$$

The left plot in Fig. 1 shows the relative echo amplitude as a function of the diffusion coefficient $D_{n}$ for three values of $n$. In each case, only the single $D_{n}$ was nonzero. For the same value of $D_{n}$, the amplitude decreases faster as $n$ increases. The right plot in this figure shows the form of the echo pulse with the $D_{1}, D_{2}$ model for a particular choice of $D_{1}, D_{2}$, and other machine parameters are taken from the RHIC values. The red curve shows the upper envelope of the pulse which is used to obtain the full width at half maximum.

\section{A. Optimum tune shift and delay time}

Analytical results for the optimum values of the tune shift and delay parameters that maximize the echo

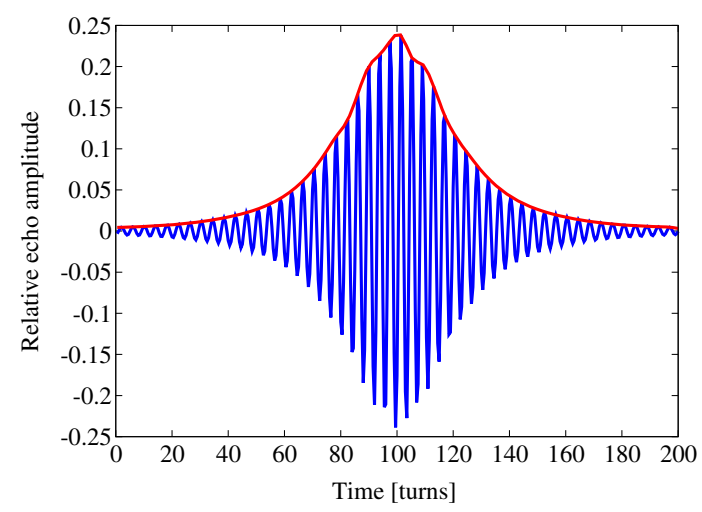

FIG. 1. Left: The echo amplitude as a function of the coefficients $D_{0}, D_{1}$, and $D_{2}$ scaled by the value $D_{\text {scale }}=2.4 \times 10^{-15} \mathrm{~m}^{2} / \mathrm{s}$. Right: Form of the echo pulse with the $D_{1}, D_{2}$ model shown in blue. The red curve outlines the upper envelope of the echo. Beam parameters in both plots were taken from Table I, except for $\mu=0.0077$. $D_{1}$ and $D_{2}$ in the right plot were set to the values $D_{1, s c}$ and $D_{2, s c}$, respectively, which are defined in Sec. III B. 
amplitude can be obtained for model 1 with diffusion coefficients $\left(d_{0}, d_{1}\right)$. As a function of the time delay, this amplitude has a maximum at a delay $\tau=\tau_{\text {opt }}$, such that the two coefficients can be related as

$$
d_{1}=\frac{1-3 d_{0} \mu_{\mathrm{fix}}^{2} \tau_{\mathrm{opt}}^{3}}{\mu_{\mathrm{fix}}^{2} \tau_{\mathrm{opt}}^{3}\left(8+3 d_{0} \mu_{\mathrm{fix}}^{2} \tau_{\mathrm{opt}}^{3}\right)} .
$$

It is understood that $\mu$ is held fixed at $\mu_{\mathrm{fix}}$ while finding the optimum delay $\tau_{\text {opt }}$. Defining $c_{\tau}=\mu_{\text {fix }}^{2} \tau_{\text {opt }}^{3}$ and substituting this into the equation for the relative amplitude, we have for the maximum amplitude obtained at the delay $\tau_{\mathrm{opt}}$

$$
\frac{\langle x\rangle_{\max }\left(\tau_{\mathrm{opt}}\right)}{\beta_{K} \theta}=\omega_{\mathrm{rev}} q \mu \tau_{\mathrm{opt}}\left[\frac{8+3 d_{0} c_{\tau}}{9}\right]^{3} \exp \left[-d_{0} c_{\tau}\right] .
$$

This equation can be solved for $d_{0}$, and subsequently $d_{1}$ can be found. Positivity of $d_{1}$ requires that the solution for $d_{0}$ obey $3 d_{0} c_{\tau} \leq 1$.

Similarly, as a function of the tune shift, the amplitude has a maximum at $\mu=\mu_{\text {opt }}$ such that

$$
d_{1}=\frac{1-2 d_{0} \mu_{\mathrm{opt}}^{2} \tau_{\mathrm{fix}}^{3}}{\mu_{\mathrm{opt}}^{2} \tau_{\mathrm{fix}}^{3}\left(5+2 d_{0} \mu_{\mathrm{opt}}^{2} \tau_{\mathrm{fix}}^{3}\right)} .
$$

Here $\tau$ is held fixed at $\tau_{\text {fix }}$ while finding the optimum in $\mu$. Defining $c_{\mu}=\mu_{\mathrm{opt}}^{2} \tau_{\text {fix }}^{3}$ and again substituting for $d_{1}$, we can write the maximum relative amplitude at $\mu_{\mathrm{opt}}$ as

$$
\frac{\langle x\rangle_{\max }\left(\mu_{\mathrm{opt}}\right)}{\beta_{K} \theta}=\omega_{\mathrm{rev}} q \mu_{\mathrm{opt}} \tau_{\mathrm{fix}}\left[\frac{5+2 d_{0} c_{\mu}}{6}\right]^{3} \exp \left[-d_{0} c_{\mu}\right] .
$$

Here $d_{1} \geq 0$ requires that the solution for $d_{0}$ obey $2 d_{0} c_{\mu} \leq 1$.

If both $\mu_{\mathrm{opt}}$ and $\tau_{\mathrm{opt}}$ are measured, then the diffusion coefficient $d_{0}$ can be found from equating the two expressions for $d_{1}$, which results in a quadratic equation for $d_{0}$ with the roots

$$
\begin{aligned}
d_{0}= & \frac{1}{12 c_{\mu} c_{\tau}}\left[2 c_{\mu}+3 c_{\tau}\right. \\
& \pm \sqrt{\left.\frac{\left(2 c_{\mu}-3 c_{\tau}\right)\left(2 c_{\mu}^{2}+67 c_{\mu} c_{\tau}+3 c_{\tau}^{2}\right)}{c_{\mu}-c_{\tau}}\right]} .
\end{aligned}
$$

Once $d_{0}$ is determined, $d_{1}$ can be determined from either of Eqs. (18) or (20). Positivity of $d_{1}$ requires that the above solution obey $d_{0} \leq 1 /\left(2 c_{\mu}\right)$ and $d_{0} \leq 1 /\left(3 c_{\tau}\right)$. This solution for both diffusion coefficients $d_{0}$ and $d_{1}$ is obtained without necessarily using the value of the echo amplitude except for recording where it has a maximum. It uses the optimum tune shift and the optimum delay and could be useful when the BPM resolution is low. However, this would require that all other beam conditions such as the dipole kick, quadrupole kick, bunch charge, etc., are kept exactly the same during both tune shift and delay scans. If this is not met, the solution given by Eq. (22) cannot be used.

For the $\left(d_{0}, d_{2}\right)$ or $\left(d_{1}, d_{2}\right)$ models discussed here, the optimum values of the tune shift and delay parameters must be found numerically.

\section{B. Echo pulse width}

In addition to the amplitude, the echo can also be characterized by the echo pulse width; e.g., the full width at half maximum (FWHM) can be chosen as a width measure.

For the model $D(J)=D_{0}+D_{1}\left(J / J_{0}\right)$, the FWHM can be found analytically from Eq. (11). We define a variable $D_{\text {up }}$ which depends on an upper limit to the pulse full width $(\Delta t)_{\mathrm{FW}}^{\mathrm{up}}$ and other parameters as follows:

$$
D_{\text {up }}=\left(\frac{\varepsilon}{\mu \omega_{\text {rev }} \tau}\right)^{2} \frac{2}{(\Delta t)_{\mathrm{FW}}^{\mathrm{up}}} .
$$

For example, with an upper limit to the pulse width of 100 turns, we have $D_{\text {up }}=2.6 \times 10^{-12} \mathrm{~m}^{2} / \mathrm{s}$. For pulse widths $\Delta t_{\mathrm{FWHM}}<(\Delta t)_{\mathrm{FW}}^{\text {up }}$ such that $\left(D_{0} / D_{\text {up }}, D_{1} / D_{\text {up }}\right) \ll 1$, we can keep terms to first order in $D_{0} / D_{\text {up }}$ and $D_{1} / D_{\text {up }}$, and we find for the FWHM

$$
\begin{aligned}
\Delta t_{\mathrm{FWHM}}= & 2 \sqrt{2^{2 / 3}-1}\left(\frac{\alpha}{\omega_{\mathrm{rev}} \mu}\right) \\
& +3\left(\frac{\alpha \tau}{\omega_{\mathrm{rev}}}\right)^{2}\left[\frac{2^{2 / 3}}{3} d_{0}+\frac{d_{1}}{\alpha}\right], \\
\alpha= & 1+\frac{1}{2} d_{1} \mu^{2} t_{1}^{3} .
\end{aligned}
$$

As we see later, we have typically $\left(D_{0} / D_{\text {up }}, D_{1} / D_{\text {up }}\right) \approx$ 0.1 , so the above assumption is satisfied for pulse widths up to 100 turns or somewhat larger. We find that the FWHM increases with increasing $D_{1}$ but very slowly with $D_{0}$ as seen in Fig. 2. When there is no diffusion, we have for the minimum FWHM

$$
\Delta t_{\mathrm{FWHM}}^{\min }=\frac{2 \sqrt{2^{2 / 3}-1}}{\omega_{\text {rev }} \mu} .
$$

In units of turns, this theoretical minimum FWHM depends only on the tune shift coefficient $\mu$. This value when compared with measured FWHM values can set limits on the tune shift parameter, as will be seen later.

For the other models with either $\left(D_{0}, D_{2}\right)$ or $\left(D_{1}, D_{2}\right)$, the time-dependent pulse shape and hence the FWHM must be found numerically. From this pulse shape, the upper 


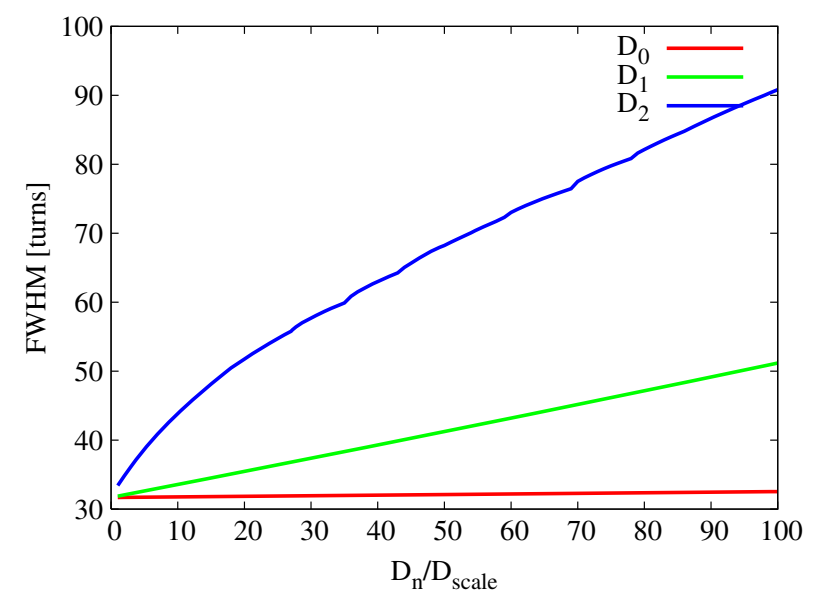

FIG. 2. FWHM as a function of the diffusion coefficients $D_{0}$,

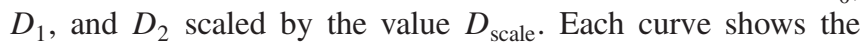
impact of the single coefficient with the others set to zero. The FWHM is calculated analytically from Eq. (24) for $D_{0}$ and $D_{1}$ and numerically for $D_{2}$. Parameters were taken from Table I, except for $\mu=0.0077$.

envelope is found numerically as an interpolating function and the FWHM then calculated from this envelope function. Figure 2 shows the dependence of the FWHM on the coefficients $D_{0}, D_{1}$, and $D_{2}$ scaled by a parameter $D_{\text {scale }}=2.4 \times 10^{-15} \mathrm{~m}^{2} / \mathrm{s}$. The FWHM increases linearly with both $D_{0}$ and $D_{1}$ but with $D_{0}$ increases by only $3 \%$ over this range. The FWHM with $D_{2}$ increases the fastest and covers the range of values obtained from the RHIC data.

\section{ANALYSIS OF RHIC DATA WITH AU IONS}

We briefly discuss the experimental procedure here; more details can be found in Ref. [12]. The echo experiments were first done with $\mathrm{Au}$ ions, later with $\mathrm{Cu}$ ions, and also with protons, all at injection energy. A special-purpose quadrupole kicker was used with a rise time of $12.8 \mu \mathrm{s}$, about one revolution time in the RHIC. The nonlinear tune shift was provided by a set of octupoles which are normally set to zero at injection, in order to observe the echoes. The initial dipole kick was delivered only in the horizontal plane by injection under a varying angle. Echoes were generated with different conditions including variable dipole and quadrupole kicks, beam intensities, tunes, different delays between the dipole kick and the quadrupole kick, and different octupole strengths. The emittance delivered to the RHIC for each species was nearly constant. While echoes were observed with each species, the most consistent echoes were obtained with the Au ions, and we will consider only those results in this article. Table I shows some of the relevant parameters for the Au ions [12].

\section{A. Emittance growth and rescaling tune shift}

In evaluating the tune shift parameter $\mu$ for calculating echo amplitudes, it is important to use the emittance following the dipole kick. The rms emittance is given by
TABLE I. Relevant RHIC parameters for the echo experiments with $\mathrm{Au}$ ions.

\begin{tabular}{lc}
\hline \hline Parameter & Nominal value \\
\hline Beam relativistic $\gamma$ & 10.52 \\
Revolution time $T_{\text {rev }}$ & $12.8 \mu \mathrm{s}$ \\
Initial emittance $\varepsilon_{0}$, unnormalized & $1.6 \times 10^{-7} \mathrm{~m}$ \\
Delay $\tau$ & 450 turns \\
Initial tune shift parameter $\mu_{0}$ & 0.0014 \\
Quadruple strength $q$ & 0.025 \\
Quadrupole rise time & $12.8 \mu \mathrm{s}$ \\
\hline \hline
\end{tabular}

$$
\begin{aligned}
\varepsilon & =\frac{1}{\beta}\left[\left\langle x^{2}\right\rangle\left\langle p^{2}\right\rangle-(\langle x p\rangle)^{2}\right]^{1 / 2} \\
& =2\left[\left\langle J \cos ^{2} \phi\right\rangle\left\langle J \sin ^{2} \phi\right\rangle-\langle J \sin \phi \cos \phi\rangle^{2}\right]^{1 / 2} .
\end{aligned}
$$

The ensemble averages are calculated using the distribution function at time $t$ after the dipole kick, which can be written in the notation of Ref. [15] as

$\psi_{2}(J, \phi, t)=\psi_{0}\left(J+\theta \sqrt{2 \beta J} \sin (\phi-\omega(J) t)+\frac{1}{2} \beta_{K} \theta^{2}\right)$,

and the averages are found from, e.g., $\left\langle J \cos ^{2} \phi\right\rangle=$ $\int d J d \phi J \cos ^{2} \phi \psi_{2}(J, \phi, t)$, etc. It can be shown that this leads to an rms emittance given by

$$
\begin{aligned}
\varepsilon(t) & =\left[\left(J_{0}+\frac{1}{2} \beta_{K} \theta^{2}\right)^{2}-A_{2}(t)^{2}\right]^{1 / 2}, \\
A_{2}(t) & =\frac{\beta_{K} \theta^{2}}{2\left(1+\Theta_{2}^{2}\right)^{3 / 2}} \exp \left[-\frac{\beta_{K} \theta^{2}}{2 J_{0}} \frac{\Theta_{2}^{2}}{1+\Theta_{2}^{2}}\right], \\
\Theta_{2} & =2 \omega^{\prime} J_{0} t .
\end{aligned}
$$

At times $t \gg \tau_{D}$, the term $A_{2} \rightarrow 0$, and we can approximate

$$
\varepsilon=J_{0}+\frac{1}{2} \beta_{K} \theta^{2}=\varepsilon_{0}\left[1+\frac{1}{2}\left(\frac{\Delta x}{\sigma_{0}}\right)^{2}\right]
$$

where $\varepsilon_{0}=J_{0}$ is the initial emittance, $\Delta x=\sqrt{\beta_{K} \beta} \theta$ is the change in the beam position at the BPM, and $\sigma_{0}=\sqrt{\beta \varepsilon_{0}}$ is the initial beam size at the BPM. The last expression in Eq. (29) has the same form as in Ref. [18]. Thus, a kick to a $3 \sigma$ amplitude results in an emittance which is 5.5 times larger than the initial emittance. We will take this as an average estimate for the emittance following the dipole kick. By definition, the tune shift parameter $\mu$ increases linearly with emittance, and hence $\mu$ increases from its nominal value of 0.0014 to 0.0077 following the dipole kick. Without this rescaling, the model cannot agree with the experimental results, as seen in the earlier analysis $[12,13]$. 


\section{B. Diffusion coefficients from optimum tune shift and delay}

The theory predicts that the maximum echo amplitude, which occurs close to the time $2 \tau$ after the dipole kick, grows indefinitely with the product $\mu \tau$ in the absence of diffusion. In the presence of any diffusion, the echo amplitude grows more slowly, reaches a maximum, and then decreases as either $\mu$ or $\tau$ is increased. In each case, the irreversible particle motion caused by the presence of diffusion reduces the amplitude of the recohering signal at the time of the echo. Here we will apply the formulas developed in Sec. II A to extract diffusion coefficients from measurements of the optimal tune shift and optimal delay.

We discuss first the analysis of the nonlinear tune shift scan done on March 11, 2004. During this scan, the quadrupole kick and delay between the dipole kick and quadrupole kick were kept constant. Octupole strengths were set to values $K_{3}=(1.5,2,2.5,5,6,7,8,9,10) \mathrm{m}^{-3}$. The nominal value was $K_{3}=7 \mathrm{~m}^{-3}$ corresponding to a nominal tune shift parameter $\mu_{0}=0.0014$ before the dipole kick. Echoes were observed for all $K_{3} \geq 2.5 \mathrm{~m}^{-3}$. The largest echoes were observed at $K_{3}=5 \mathrm{~m}^{-3}$, which corresponds to a nominal tune shift parameter $\mu=0.001$, while the rescaled tune shift value is $\mu_{\mathrm{opt}}=0.0055$.

For the $D_{0}, D_{1}$ model, the starting solutions were obtained by solving Eqs. (20) and (21). These yielded $d_{0}=$ $2.245 \times 10^{10} \mathrm{~s}^{-3}$ and $d_{1}=2.435 \times 10^{10} \mathrm{~s}^{-3}$, which lead to $D_{0}=1.08 \times 10^{-13} \mathrm{~m}^{2} / \mathrm{s}$ and $D_{1}=1.17 \times 10^{-13} \mathrm{~m}^{2} / \mathrm{s}$, respectively. These found values for $\left(D_{0}, D_{1}\right)$ yield a maximum at $\mu_{\text {opt }}=0.0055$ by design, but the amplitude values decrease more slowly with $\mu$ than the data. To improve the fit with the data, a numerical fitting was done (using Mathematica [19]) to the data with the model shown in Eq. (11). These yielded $D_{0}=1.62 \times 10^{-13} \mathrm{~m}^{2} / \mathrm{s}$ and $D_{1}=1.19 \times 10^{-13} \mathrm{~m}^{2} / \mathrm{s}$ and led to a better fit with all the data. These values for $D_{0}$ and $D_{1}$ were labeled as $D_{0, s c}$ and $D_{1, s c}$, respectively, and subsequent values were scaled by these values for convenience. With both the $\left(D_{0}, D_{2}\right)$ and the $\left(D_{1}, D_{2}\right)$ models, a least-squares minimization was done to fit the data against the respective models for the amplitude. The fit for $D_{2}$ from the $\left(D_{0}, D_{2}\right)$ model was similarly labeled as $D_{2, s c}$. The resulting fits and the data are shown in Fig. 3. The values of the coefficients are shown in Table II. Relative to the previous comparison of theory with experimental data (cf. Fig. 4 in Ref. [12]), these fits show a significant improvement. Of the three models, the best fit with the lowest chi squared is seen with the $\left(D_{0}, D_{2}\right)$ model with the next best being the $\left(D_{1}, D_{2}\right)$ model. However, the models are fairly close, and no model can be ruled out based on these data.

On a later day (March 17, 2004), the delay $\tau$ between the dipole kick and the quadrupole kick was varied with values $(450,500,550,600,900)$ turns. Echoes were

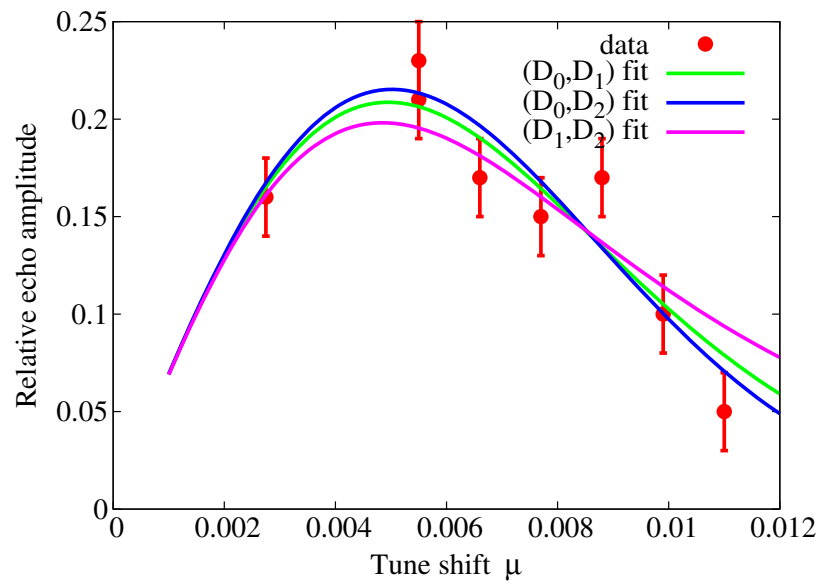

FIG. 3. Comparison of the echo amplitude vs the tune shift strength scan. The data are shown in red with error bars, while the fits shown are with the three models for the diffusion coefficients discussed in the text.

observed only at the first three values of the delay. In all, six echoes were observed with the largest amplitudes at 450 turns. The quadrupole kick strength, the octupole strengths, and the tunes were kept constant. We will use this limited data set to obtain the diffusion coefficients from the delay scan.

For the $\left(D_{0}, D_{1}\right)$ model, we start by solving Eqs. (18) and (19) for the coefficients from the echo amplitude and the value of the optimum delay $\tau_{\text {opt }}$. Again, better fits to the data are obtained by a least-squares minimization, which is also the procedure for the other two models. Table II shows the best fit values with this delay scan. Compared to the values from the tune shift scan, the coefficients for the same model are within a factor of 2 from this delay scan. Some of the variation in the values between the scans can be due to different beam conditions on the two days such as bunch intensities and machine tunes. However, the uncertainties associated with these values are large, since there were too few data points. Figure 4 shows the comparison of the fitted models with the data. Again, all three models show similar goodness of fits with the best fit (minimum chi squared) obtained with the $\left(D_{0}, D_{2}\right)$ model, but all chi squared values are close. All models show that the relative echo amplitude reaches a maximum at around 390 turns, which is less than the minimum delay of 450 turns used in the experiment.

TABLE II. Comparison of the diffusion coefficients from the tune shift and delay scans. All diffusion coefficients are in units of $10^{-13} \mathrm{~m}^{2} / \mathrm{s}$.

\begin{tabular}{lcc}
\hline \hline Model & Tune shift scan & Delay scan \\
\hline$D_{0} / D_{1}$ & $1.6 / 1.3$ & $0.65 / 1.3$ \\
$D_{0} / D_{2}$ & $1.9 / 0.025$ & $3.7 / 0.015$ \\
$D_{1} / D_{2}$ & $2.3 / 0.025$ & $1.9 / 0.013$ \\
\hline \hline
\end{tabular}




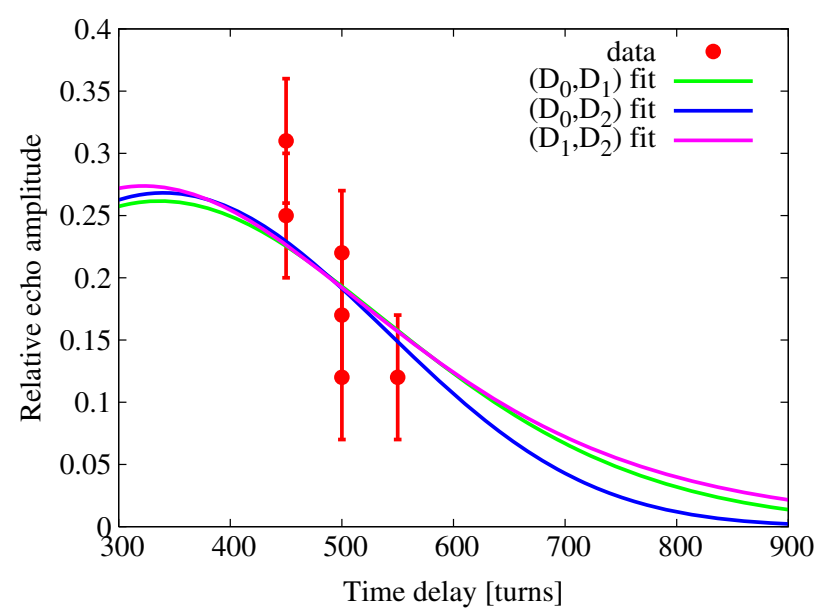

FIG. 4. Comparison of the echo amplitude vs the time delay between the dipole and quadrupole kicks. The data are shown with error bars, while the fits are shown with the three models discussed in the text.

\section{Diffusion coefficients from the echo amplitude and the FWHM}

The above analysis has shown that all three models are viable candidates in describing the data dependence on either the tune shift or the delay. We now use turn by turn (TBT) data to fit both the echo amplitude and the echo pulse width with each model. Ten such data sets could be retrieved from the 2004 measurements. In this TBT set, the initial dipole kick and bunch charge varied, but the other parameters including the quadrupole kick strength, tunes, delay, and octupole strengths were kept constant. Figures 5 and 6 show two examples from this set, one with a clean echo pulse and the other where the beam centroid takes a longer time to decohere after the initial kick and the echo pulse is also much wider. Some of the more distorted signals could be due to oscillations from off-axis injection and could partly be due to a fourth-order resonance and slightly higher bunch charge. For each data set, an interpolating function was found to fit the upper envelope

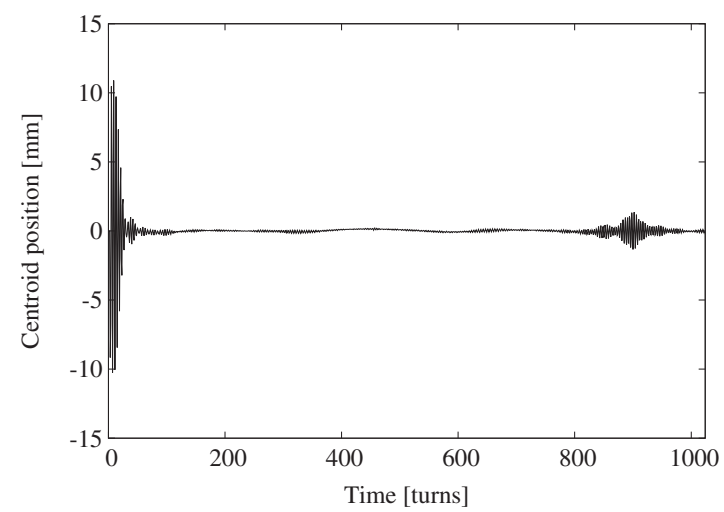

of the echo pulse, and the FWHM was extracted from this interpolating function. Using the value of the rescaled tune shift parameter $\mu=0.077$, the minimum theoretical value of the FWHM without diffusion, using Eq. (25), is 32 turns. This is consistent with the minimum FWHM with diffusion from the data set, which is 37 turns. The bare tune shift parameter of $\mu_{0}=0.0014$ would have predicted a minimum FWHM of 160 turns, much larger than any FWHM value measured.

Figure 7 shows the FWHM plotted as a function of the number of particles per bunch. This figure shows that the FWHM fell into three distinct clusters, because the bunch charge varied around three values. Except for the two outlier points labeled as 1 and 2, all other points show that the FWHM increases with charge. These other points are fit to a power law curve

$$
\operatorname{FWHM}(N)=\Delta t_{\mathrm{FWHM}}^{\min }+a N^{p}
$$

where $\Delta t_{\mathrm{FWHM}}^{\min }$ is the minimum FWHM from Eq. (25), $N$ is the number of particles per bunch, and $(a, p)$ are the fit parameters. The fit shows that the exponent is $p=2.002$, so the FWHM increases quadratically with the charge. Since the tune shift, delay, and tune were kept constant during these measurements, the outlier points show that the FWHM values may depend on other parameters, such as the initial dipole kick amplitude.

We now solve for two diffusion coefficients using the relative echo amplitude and the FWHM. For the $\left(D_{0}, D_{1}\right)$ model, the FWHM can be found analytically, as shown in Eq. (24). The $d_{0}$ coefficient can be written as a function of the echo amplitude and $d_{1}$ using the echo amplitude equation (11) as

$$
d_{0}=-\frac{1}{\mu^{2} \tau^{3}} \ln \left[\frac{\langle x\rangle_{\mathrm{rel}}^{\mathrm{amp}}}{2 \pi q \mu N_{\text {delay }}}\left(1+\mu^{2} \tau^{3} d_{1}\right)^{3 / 2}\right]
$$

where $\langle x\rangle_{\text {rel }}^{\mathrm{amp}}=\langle x\rangle^{\mathrm{amp}} /\left(\beta_{K} \theta\right)$ is the relative echo amplitude in terms of the dipole kick and $N_{\text {delay }}=\tau / T_{\text {rev }}$ is the

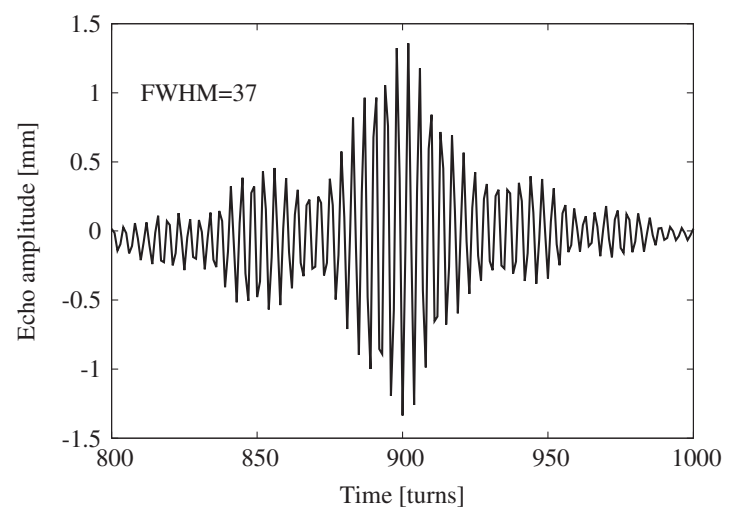

FIG. 5. The entire centroid position turn by turn (left) and the echo pulse isolated (right) for the data with the shortest FWHM. Here the centroid decoheres cleanly after the dipole kick. The quadrupole kick was applied at turn 450. 

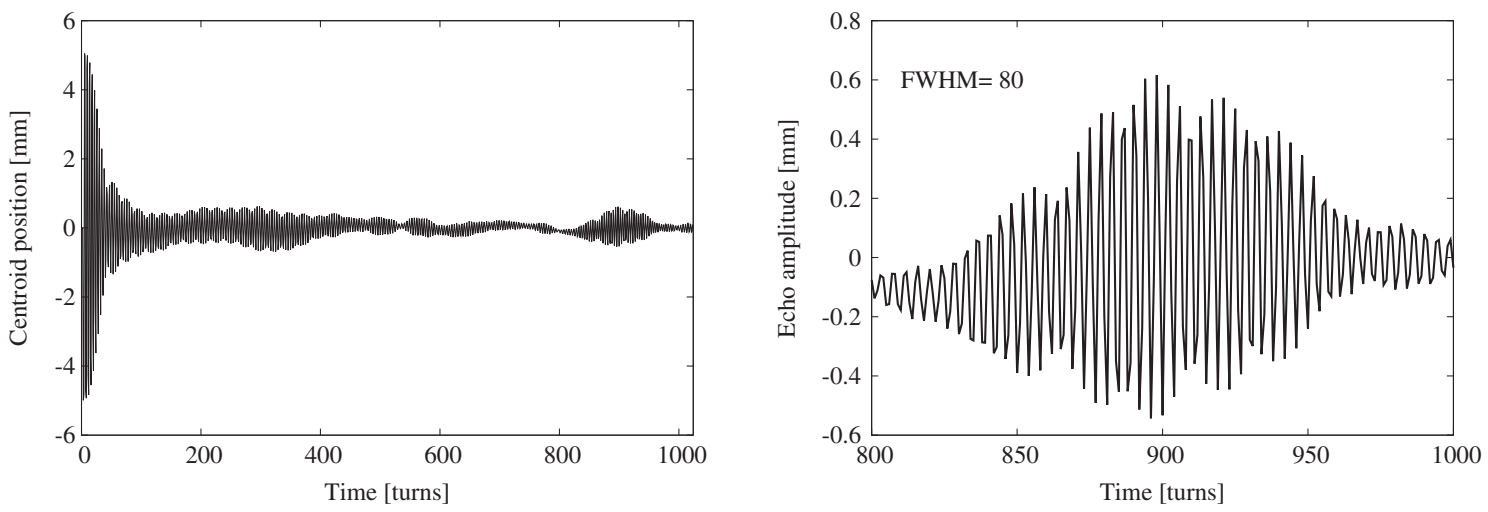

FIG. 6. The entire centroid position turn by turn (left) and the echo pulse isolated (right) for the data with the largest FWHM. Notice the much larger and longer ringing of the centroid after the dipole kick. The quadrupole kick was applied again at turn 450.

delay in units of turns. The positivity of $d_{0}$ implies an upper limit to $d_{1}$ as

$$
d_{1}^{\max }=\frac{1}{\mu^{2} \tau^{3}}\left[\left(\frac{2 \pi q \mu N_{\text {delay }}}{\langle x\rangle_{\text {rel }}^{\text {amp }}}\right)^{1 / 3}-1\right] .
$$

The value of $d_{1}$ can be found by numerically solving Eq. (24) for the FWHM with $d_{0}$ substituted from Eq. (31). We find that this $\left(D_{0}, D_{1}\right)$ model yields positive $d_{0}$ coefficients in only four of the ten cases. We conclude, therefore, that the $D_{0}, D_{1}$ model is not well suited for this data.

With the $\left(D_{0}, D_{2}\right)$ model, the $d_{0}$ coefficient can again be found analytically as a function of the echo amplitude and $d_{1}$ using

$d_{0}=-\frac{1}{\mu^{2} \tau^{3}} \ln \left[\frac{\langle x\rangle_{\text {rel }}^{\mathrm{amp}}}{\pi q \mu N_{\text {delay }}} \frac{1}{\operatorname{Im}\left[e^{i \Phi_{0}\left(T_{\text {rev }}\right)} H_{02}\left(T_{\text {rev }}\right)\right.}\right]$,

where $H_{02}$ is defined in Eq. (13). We find again that no solutions with positive $D_{0}$ can be found in all cases with

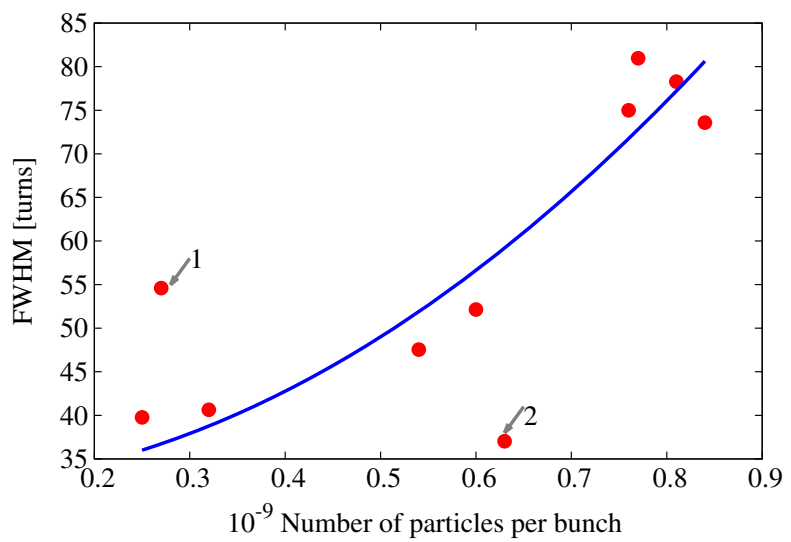

FIG. 7. FWHM vs the number of particles per bunch. Except for the points labeled 1 and 2, all the other data points show an increasing FWHM with bunch charge. The blue curve shows a quadratic fit through these points.
FWHM $>70$ turns. Even in other cases where the solutions can be found, the values of $D_{2}$ are significantly larger than the values found in the previous sections and, hence, appear to be in a disconnected region of the parameter space. Since $D_{0}$ has little impact on the FWHM (see Fig. 2), in both the $\left(D_{0}, D_{1}\right)$ and $\left(D_{0}, D_{2}\right)$ models, large values of the FWHM can make $D_{1}$ or $D_{2}$ large which then require a negative $D_{0}$ to satisfy the amplitude condition. Thus, fitting the models to both the amplitude and FWHM rules out the models with $D_{0}$.

In the case of the $D_{1}, D_{2}$ model, neither coefficient can be found analytically from the amplitude equation. Instead, the amplitude and the FWHM equations must be solved numerically. Figure 8 shows the forms of the function $\operatorname{ampl}\left(d_{1}, d_{2}\right)$ and fwhm $\left(d_{1}, d_{2}\right)$. Also shown are the intersections of these surfaces with the plane of constant amplitude or FWHM value, respectively. In each case, the intersection of the surface with the plane determines a curve of solutions for that equation. The intersection of the two curves in the $d_{1}, d_{2}$ plane would determine the required solution for given values of the amplitude and FWHM. In this figure, the values of $d_{1}$ and $d_{2}$ are scaled by $d_{1, s c}$ and $d_{2, s c}$, which are obtained from $D_{1, s c}$ and $D_{2, s c}$ using Eq. (9). These plots demonstrate that, for the range of measured values of the echo amplitude and the FWHM, solutions for the diffusion coefficients exist in the range $0 \leq\left(d_{1} / d_{1, s c}, d_{2} / d_{2, s c}\right) \leq 8$.

It turns out to be easier to do a least-squares minimization to find the solution. Here we define the $\chi^{2}$ function as

$$
\begin{aligned}
\chi^{2}= & \left(\frac{\operatorname{ampl}\left(d_{1}, d_{2}\right)-\mathrm{ampl}_{\mathrm{data}}}{\sigma_{\mathrm{ampl}}}\right)^{2} \\
& +\left(\frac{\mathrm{fwhm}\left(d_{1}, d_{2}\right)-\mathrm{fwhm}_{\mathrm{data}}}{\sigma_{\mathrm{FWHM}}}\right)^{2},
\end{aligned}
$$

where $\operatorname{ampl}\left(d_{1}, d_{2}\right)$ and $\operatorname{fwhm}\left(d_{1}, d_{2}\right)$ are the amplitude function [from Eq. (16)] and the FWHM function defined numerically, respectively, and $\sigma_{\mathrm{ampl}}=0.05$ and $\sigma_{\mathrm{FWHM}}=2$ are the estimated uncertainties in the two data variables. 

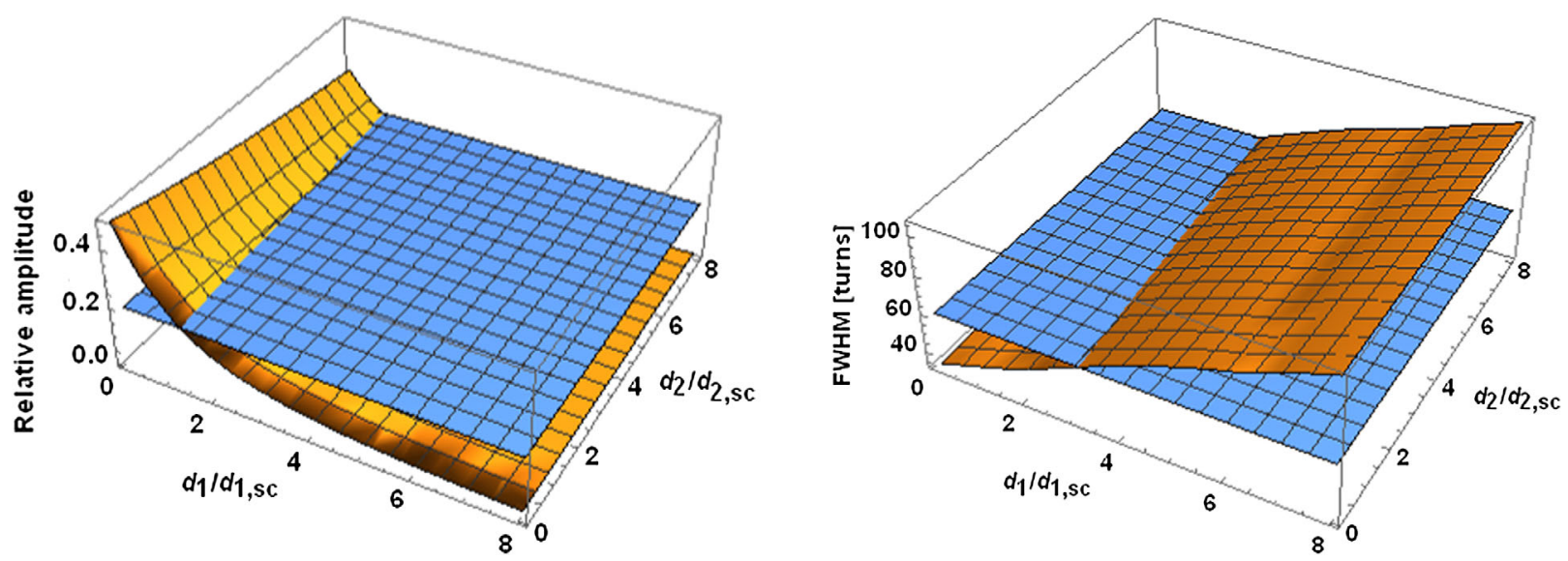

FIG. 8. Left: Relative echo amplitude (in brown) as a function of the scaled diffusion coefficients $d_{1} / d_{1, s c}$ and $d_{2} / d_{2, s c}$ intersected by a plane (in blue) of a particular relative amplitude value, here chosen to be 0.2 . The intersection defines the family of solutions for $\left(d_{1}, d_{2}\right)$ at this amplitude. Right: The FWHM (in brown) as a function of the same scaled variables and the plane (in blue) at a constant FWHM, here chosen to be 60 turns. Again, the intersection defines the family of solutions for the FWHM equation.

This least-squares method turns out to be efficient and leads to positive solutions for $d_{1}$ and $d_{2}$ in all cases. Table III shows the values of the diffusion coefficients in these cases. We observe that these values are close to the values of $D_{1}$ found from the optimal tune shift and delay measurements shown in Table II. The $D_{2}$ values differ by an order of magnitude in the two tables, but, considering that the delay and tune shift scan methods for the amplitude are less sensitive to $D_{2}$ and also from the larger number of data points in the FWHM analysis, we expect the values in Table III to be more accurate. In most cases, the $D_{1}$ coefficient is an order of magnitude greater than $D_{2}$. The single exception (row 2 of this table) corresponds to the outlier point labeled 1 in Fig. 7. As a function of charge, $D_{1}$ increases, while $D_{2}$ appears to be independent of the charge.

\section{Diffusion dependence on bunch charge}

We focus now on the $\left(D_{1}, D_{2}\right)$ model, which is the only one of those studied that can describe both the amplitude

TABLE III. Diffusion coefficients $\left(D_{1}, D_{2}\right)$ found using the amplitude and the FWHM values from the turn by turn data.

\begin{tabular}{lcccc}
\hline \hline $\begin{array}{l}\text { Particles per } \\
\text { bunch }\left[10^{9}\right]\end{array}$ & $\begin{array}{c}\text { Rel. } \\
\text { ampl. }\end{array}$ & $\begin{array}{c}\text { FWHM } \\
\text { [turns] }\end{array}$ & $D_{1}\left[10^{-13} \mathrm{~m}^{2} / \mathrm{s}\right]$ & $D_{2}\left[10^{-13} \mathrm{~m}^{2} / \mathrm{s}\right]$ \\
\hline 0.25 & 0.245 & 39.8 & 1.28 & 0.0030 \\
0.27 & 0.225 & 54.6 & 0.13 & 0.51 \\
0.32 & 0.160 & 40.6 & 1.49 & 0.32 \\
0.54 & 0.127 & 47.5 & 2.00 & 0.28 \\
0.6 & 0.142 & 52.1 & 1.98 & 0.21 \\
0.63 & 0.125 & 37.0 & 1.98 & 0.30 \\
0.76 & 0.114 & 75.0 & 2.53 & 0.24 \\
0.77 & 0.122 & 81.0 & 2.18 & 0.24 \\
0.81 & 0.110 & 78.3 & 2.53 & 0.24 \\
0.84 & 0.0998 & 73.6 & 2.53 & 0.24 \\
\hline \hline
\end{tabular}

and the pulse width of the echo. During the measurements on March 17, 2004, an intensity scan was done with all other parameters kept constant. While the turn by turn data from that scan are not easily accessible, the echo amplitudes are available with 27 data points. These data can be used to measure the diffusion coefficients as a function of the bunch charge.

Both $\left(D_{1}, D_{2}\right)$ coefficients can be found by a leastsquares minimization of the fit to the amplitude. This process allows a determination of $\left(D_{1}, D_{2}\right)$ as a function of the charge. The left plot in Fig. 9 shows the $D_{1}$ values found and a linear fit to the values. This confirms the behavior seen in the previous section but now with a larger data set. Similarly as earlier, the $D_{2}$ values are nearly independent of the charge. We can parameterize the echo amplitude's dependence on the bunch charge via these fits for $D_{1}$ and $D_{2}$ and the amplitude equation (16). The linear fit yields $d_{1} / d_{1, s c}=0.42+2.78 N$, where $N$ is the number of particles per bunch in units of $10^{9}$, while for $d_{2}$ we take the mean value over this set, $d_{2} / d_{2, s c}=6.24$. The right plot in Fig. 9 shows the measured echo amplitudes (in red) as a function of the number of particles per bunch and also the calculated amplitude (in blue) from these fits for $\left(D_{1}, D_{2}\right)$. The measured echo amplitude decreases with increasing charge, and this trend is well reproduced by the theoretical amplitude function. This is a consistency check and is to be expected, since the linear fit for $d_{1}$ and constant for $d_{2}$ were obtained from the data set. The comparison in Fig. 9 shows that we can parameterize the diffusion coefficients as

$$
D(J)=\left[a_{10}+a_{11} N\right]\left(\frac{J}{J_{0}}\right)+a_{20}\left(\frac{J}{J_{0}}\right)^{2},
$$

where $a_{10}, a_{11}$, and $a_{20}$ are functions of machine and beam parameters such as the nonlinearity, tunes, emittance, etc., but independent of the bunch charge. 

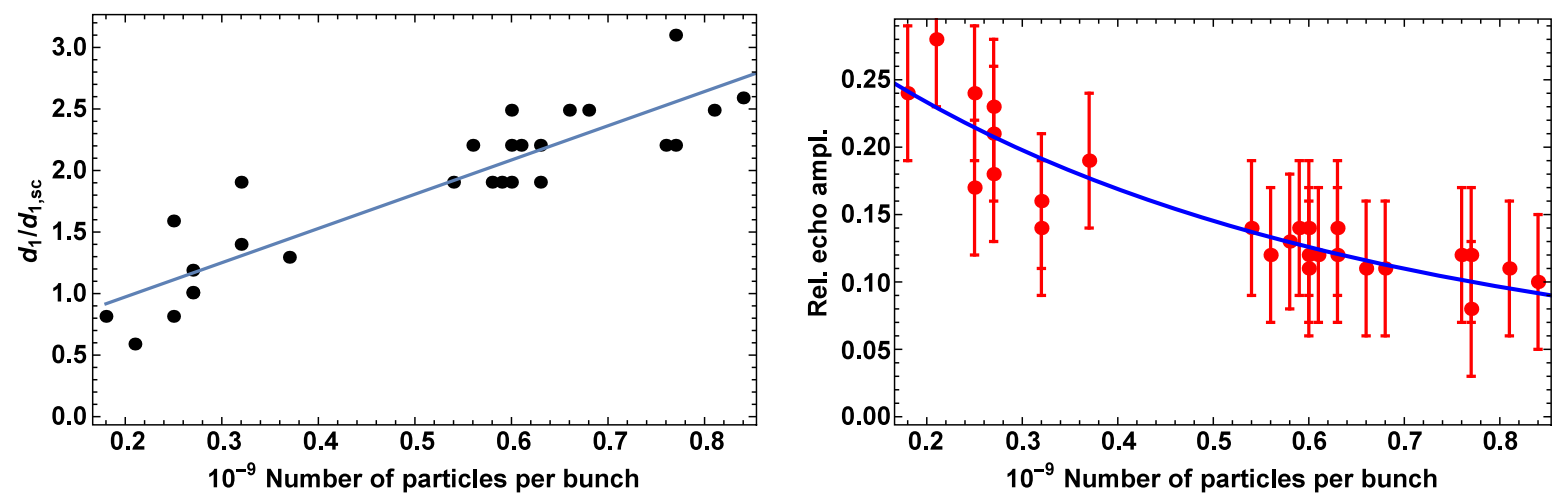

FIG. 9. Left: Calculated $d_{1}$ values as a function of the number of particles per bunch and the linear fit to the values. Right: Measured relative echo amplitude (red) at different intensities and compared with the best fit curve (blue) with $\left(D_{1}, D_{2}\right)$, with D1 from the linear fit in the left plot and D2 independent of the bunch charge.

Space charge effects and intrabeam scattering (IBS) are the dominant sources of particle diffusion for heavy ions such as Au in the RHIC, at injection energy. The incoherent space charge and IBS-induced diffusion and emittance growth depend linearly on the charge, and our analysis confirms that the leading diffusion coefficient $D_{1}$ increases linearly with the charge. The coefficient $D_{2}$ is likely to be determined by diffusion from single particle nonlinear dynamics processes.

In the above analysis, we have neglected the effect of wakefields on the echo formation. Their impact on the calculations above is not likely to be significant. As seen in Figs. 5 and 6 and generally true for the available turn by turn data, the centroid response after the dipole kick is cleaner and the relative echo amplitude is larger with the larger amplitude kick. This would likely not be the case if the effects of the transverse wake were significant. Instead, effects due to injection oscillations and fourth-order resonance which shows up at intermediate amplitudes are the likely reason for the response seen in Fig. 6. In addition, the effect of wakefields would be visible in a change in the decoherence time with intensity. An analysis of the intensity scan data shows no correlation between the decoherence time and the bunch intensity.

\section{MEAN ESCAPE TIME}

One useful time scale that can be extracted from the diffusion coefficients is the mean escape time $t_{\text {esc }}$ associated with probabilistic processes [20]. This time, also known as the mean first passage time, is the mean time taken (averaging over many realizations of the process) for a particle to escape from a certain region defined by a boundary. It was shown in Ref. [21] that, in the case that $D(J)=D_{1}\left(J / J_{0}\right)$, the time-dependent density distribution solution $\psi(J, t)$ to the diffusion equation leads to a beam lifetime $t_{L}$ which is close to the escape time $t_{\text {esc }}$ estimate. Defining $t_{L}=-N(t) /(d N / d t)$, where $N(t)=\int \psi(J, t) d J$ is the particle number, it was shown that

$$
t_{L} \approx 0.7 \frac{J_{A} J_{0}}{D_{1}}, \quad t_{\mathrm{esc}}=\frac{J_{A} J_{0}}{D_{1}},
$$

where $J_{A}$ is the action at the absorbing boundary. We will assume that the mean escape time is also a useful beam relevant time scale when $D(J)=D_{1}\left(J / J_{0}\right)+D_{2}\left(J / J_{0}\right)^{2}$.

The mean escape time from an action $J$ to an absorbing boundary at action $J_{A}$ is given by

$$
\begin{aligned}
t_{\mathrm{esc}}(J) & =\int_{J}^{J_{A}} d J \frac{J}{D(J)}=\int_{J}^{J_{A}} d J \frac{J}{D_{1}\left(J / J_{0}\right)+D_{2}\left(J / J_{0}\right)^{2}} \\
& =\frac{J_{0}^{2}}{D_{2}} \ln \left[\frac{D_{1}+D_{2}\left(J_{A} / J_{0}\right)}{D_{1}+D_{2}\left(J / J_{0}\right)}\right] .
\end{aligned}
$$

This is the mean escape time for particles initially at a single action $J$ to reach the aperture at action $J_{A}$ due to diffusion. A parameter describing the escape time for the beam can be obtained by averaging this over the initial beam distribution $\psi_{0}(J)$, which yields

$$
\begin{aligned}
\left\langle t_{\mathrm{esc}}\right\rangle & =\frac{J_{0}}{D_{2}} \int_{0}^{\infty} d J \exp \left[-\frac{J}{J_{0}}\right] \ln \left[\frac{D_{1}+D_{2}\left(J_{A} / J_{0}\right)}{D_{1}+D_{2}\left(J / J_{0}\right)}\right] \\
& =\frac{J_{0}^{2}}{D_{2}}\left[\ln \left(\frac{D_{1}}{D_{2}}+\frac{J_{A}}{J_{0}}\right)-\ln \frac{D_{1}}{D_{2}}-e^{D_{1} / D_{2}} \Gamma\left(0, \frac{D_{1}}{D_{2}}\right)\right] \\
& \equiv \frac{J_{0}^{2}}{D_{2}} A_{F},
\end{aligned}
$$

where $\Gamma(0, z)$ is the incomplete Gamma function and we have assumed $D_{2} \neq 0$. The dimensionless amplifying factor $A_{F}$, defined by the terms in square brackets, depends on only the ratios $D_{1} / D_{2}$ and $J_{A} / J_{0}$. Figure 10 shows the dependence of the dimensionless terms on $D_{1} / D_{2}$ for three values of $J_{A} / J_{0}$ corresponding to apertures at $(6,10,12) \sigma$, respectively. For $D_{1} / D_{2} \simeq 10, A_{F}$ is of the order of unity. Hence, the mean escape time is determined primarily by $J_{0}^{2} / D_{2}$. In the case that $D_{2}=0$, the time scale would be determined by $J_{0} J_{A} / D_{1}$. With $J_{0}=1.6 \times 10^{-7} \mathrm{~m}$, and 


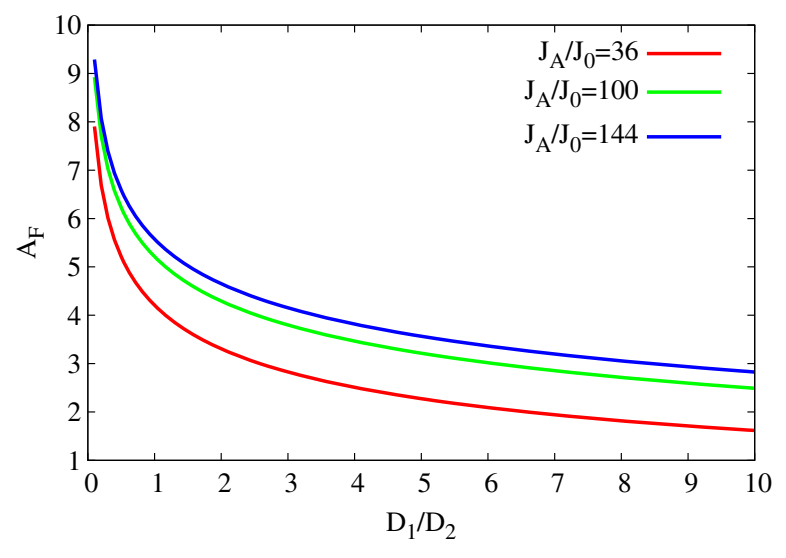

FIG. 10. Dependence of $A_{F}$, defined in Eq. (38), on the ratio of diffusion coefficients $D_{1} / D_{2}$ for three values of the ratio of the action at the absorbing aperture $J_{A}$ to the initial emittance $J_{0}$.

taking a representative value $D_{2}=0.25 \times 10^{-13} \mathrm{~m}^{2} / \mathrm{s}$ from Table III, we have $\left\langle t_{\text {esc }}\right\rangle \approx 1 \mathrm{~s}$. While this time is extremely short, it corresponds to the lifetime of a beam at large amplitudes and not to a beam circulating on the nominal closed orbit. Observations in the RHIC did show that the lifetimes of kicked beams were significantly smaller compared to those for beams not kicked. However, the early losses of the kicked beams were dominated by scraping at aperture restrictions, so there is no straightforward way to determine the contribution of diffusion to those lifetimes. Nevertheless, the diffusion coefficients and the associated time scales should be useful for relative measures of beam growth and particle loss. As an example, it could be useful in IOTA to quickly distinguish between lattices with different degrees of integrability. If echoes can be generated by smallamplitude kicks, then the calculated diffusion coefficients and the time scales would be more representative of beam behavior under nominal conditions. Determining the diffusion coefficients may require different parameterizations of $D(J)$ at small and large amplitudes, as seen, for example, in Ref. [16].

\section{SUMMARY}

In this article, we revisited earlier observations of transverse beam echoes in the RHIC to extract diffusion coefficients from those measurements. We considered three models for the action dependence of the diffusion coefficients: $D(J)=D_{0}+D_{1}\left(J / J_{0}\right), D(J)=D_{0}+D_{2}\left(J / J_{0}\right)^{2}$, and $D(J)=D_{1}\left(J / J_{0}\right)+D_{2}\left(J / J_{0}\right)^{2}$. All three models were found to adequately describe the echo amplitudes measured during scans of the nonlinear tune shift and the delay between the dipole and quadrupole kicks. Next, turn by turn data were used to extract both the amplitude and the FWHM of the pulse width. Here both models with $D_{0}$ do not describe the data with larger pulse widths, so the only model that successfully describes both the amplitude and the FWHM data is the $\left(D_{1}, D_{2}\right)$ model. We find that $D_{1}$ is an order of magnitude larger than $D_{2}$ in most cases; it increases linearly with the charge, while $D_{2}$ is nearly independent of the charge. Using these charge dependencies, the $\left(D_{1}, D_{2}\right)$ model also adequately describes another set of data where the echo amplitudes were measured as a function of the charge.

These results show that transverse echoes can indeed be used to measure transverse beam diffusion in existing and future hadron synchrotrons. We make some observations on requirements for future measurements. The diffusion measurements require good control of several machine and beam parameters such as the initial dipole kick, the quadrupole kick, machine nonlinearity, tunes, and beam emittance, to name the most important. Injection oscillations can strongly influence the echo amplitude and pulse shape, so these need to be controlled to the extent possible. Alternatively, if available, a fast dipole kicker in the ring would be preferable to initiate the echo. In such a case, a transverse damper can damp initial oscillations and then be turned off before the dipole kicker is used. While the echo amplitude variation with scans of the tune shift and time delay are useful, detailed analysis of the turn by turn data yields more information. As an example of this, we found that the FWHM scales quadratically with the charge and therefore is more sensitive to intensity changes than the echo amplitude. The proximity of resonances can also spoil echoes, so the tunes and the dipole kick amplitudes need to be chosen carefully as well.

\section{ACKNOWLEDGMENTS}

Fermilab is operated by Fermi Research Alliance, LLC under U.S. Department of Energy Contract No. DE-AC0207CH11359. Brookhaven National Laboratory is operated by Brookhaven Science Associates, LLC under U.S. Department of Energy Contract No. DE-AC0298CH10886.

[1] K. Mess and M. Seidel, Collimators as diagnostic tools in the proton machine of HERA, Nucl. Instrum. Methods Phys. Res., Sect. A 351, 279 (1994).

[2] W. Fischer, M. Giovannozzi, and F. Schmidt, Dynamic aperture experiment at a synchrotron, Phys. Rev. E 55, 3507 (1997).

[3] R. P. Fliller III, A. Drees, D. Gassner, G. McIntyre, S. Peggs, and D. Trbojevic, Beam diffusion measurement in RHIC, in Proceedings of the 2003 Particle Accelerator Conference, Portland, OR (IEEE, New York, 2003), p. 2904.

[4] G. Valentino, R. Assmann, R. Bruce, F. Burkart, V. Previtali, S. Redaelli, B. Salvachua, G. Stancari, and A. Valishev, Beam diffusion measurements using collimator scans in the LHC, Phys. Rev. ST Accel. Beams 16, 021003 (2013).

[5] G. Stancari, Measurements of beam halo diffusion and population density in the Tevatron and in the Large Hadron Collider, in Proceedings of HB2014, 54th ICFA Advanced 
Beam Dynamics Workshop on High-Intensity, High Brightness and High Power Hadron Beams, 2014 (Michigan State University, East Lansing, 2014), p. 294.

[6] G. V. Stupakov, Report No. SSCL-579, 1992.

[7] G. V. Stupakov and S. K. Kaufmann, Echo effect in accelerators, AIP Conf. Proc. 326, 522 (1995); Report No. SSCL-587, 1992.

[8] G. V. Stupakov and A. W. Chao, Effect of diffusion on bunched beam echo, in Proceedings of the Particle Accelerator Conference, Vancouver, BC, Canada, 1997 (IEEE, New York, 1997), p. 1834.

[9] L. K. Spentzouris, J.-F. Ostiguy, and P. L. Colestock, Direct Measurement of Diffusion Rates in High Energy Synchrotrons Using Longitudinal Beam Echoes, Phys. Rev. Lett. 76, 620 (1996).

[10] O. Bruning, T. Linnecar, F. Ruggiero, W. Scandale, E. Shaposhnikova, and D. Stellfeld, Beam echoes in the CERN SPS, in Proceedings of the Particle Accelerator Conference, Vancouver, BC, Canada, 1997 (IEEE, New York, 1997), p. 1816.

[11] G. Arduini, F. Ruggiero, F. Zimmermann, and M. ZorzanoMier, Report No. CERN-SL-Note-2000-048-MD, 2000.

[12] W. Fischer, T. Satogta, and R. Tomas, Measurement of transverse echoes in RHIC, in Proceedings of the 21st Particle Accelerator Conference, Knoxville, TN, 2005 (IEEE, Piscataway, NJ, 2005), p. 1955.
[13] S. Sorge, O. Boine-Frankenheim, and W. Fischer, Analysis of measured transverse beam echoes in RHIC, in Proceedings of ICAP06, International Computational Accelerator Physics, Chamonix, France, 2006 (2006), p. 234.

[14] S. Nagaitsev, A. Valishev, V. V. Danilov, and D. N. Shatilov, Design and simulation of IOTA-a novel concept of Integrable Optics Test Accelerator, in Proceedings of the 3rd International Particle Accelerator Conference, New Orleans, LA, 2012 (IEEE, Piscataway, NJ, 2012), p. 16.

[15] A. W. Chao, lecture notes at http://www.slac.stanford.edu/ $\sim$ achao/lecturenotes.html.

[16] T. Chen et al., Measurements of a Hamiltonian System and Their Description by a Diffusive Model, Phys. Rev. Lett. 68, 33 (1992).

[17] F. Zimmermann, Transverse proton diffusion, Part. Accel. 49, 67 (1995).

[18] D. Edwards and M. J. Syphers, Introduction to the Physics of High Energy Accelerators (Wiley, New York, 1993).

[19] Wolfram Research Inc., Mathematica, Version 10.4, Champaign, IL, 2016.

[20] C. W. Gardiner, Handbook of Stochastic Methods (Springer, New York, 1985).

[21] T. Sen, Diffusion dynamics in a Tevatron store, J. Instrum. 6, P10017 (2011). 